\title{
Front Matter: Volume 11522
}

, "Front Matter: Volume 11522," Proc. SPIE 11522, Optical Manipulation and Structured Materials Conference 2020, 1152201 (15 June 2020); doi: $10.1117 / 12.2574114$

SPIE Event: SPIE Technologies and Applications of Structured Light, 2020, SPIE. Yokohama, Japan 


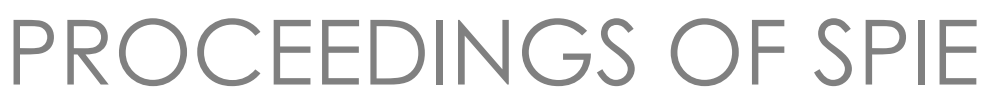

\section{Optical Manipulation and Structured Materials Conference 2020}

Takashige Omatsu

Kishan Dholakia

Hajime Ishihara

Keiji Sasaki

Editors

Yokohama, Japan

20-24 April 2020

Sponsored and Published by

SPIE 
The papers in this volume were part of the technical conference cited on the cover and title page. Papers were selected and subject to review by the editors and conference program committee. Some conference presentations may not be available for publication. Additional papers and presentation recordings may be available online in the SPIE Digital Library at SPIEDigitalLibrary.org.

The papers reflect the work and thoughts of the authors and are published herein as submitted. The publisher is not responsible for the validity of the information or for any outcomes resulting from reliance thereon.

Please use the following format to cite material from these proceedings:

Author(s), "Title of Paper," in Optical Manipulation and Structured Materials Conference 2020, edited by T. Omatsu, K. Dholakia, H. Ishihara, K. Sasaki, Proceedings of SPIE Vol. 11522 (SPIE, Bellingham, WA, 2020) Seven-digit Article CID Number.

ISSN: 0277-786X

ISSN: 1996-756X (electronic)

ISBN: 9781510638518

ISBN: 9781510638525 (electronic)

Published by

SPIE

P.O. Box 10, Bellingham, Washington 98227-0010 USA

Telephone +1 3606763290 (Pacific Time) · Fax +1 3606471445

SPIE.org

Copyright (c) 2020, Society of Photo-Optical Instrumentation Engineers.

Copying of material in this book for internal or personal use, or for the internal or personal use of specific clients, beyond the fair use provisions granted by the U.S. Copyright Law is authorized by SPIE subject to payment of copying fees. The Transactional Reporting Service base fee for this volume is $\$ 21.00$ per article (or portion thereof), which should be paid directly to the Copyright Clearance Center (CCC), 222 Rosewood Drive, Danvers, MA 01923. Payment may also be made electronically through CCC Online at copyright.com. Other copying for republication, resale, advertising or promotion, or any form of systematic or multiple reproduction of any material in this book is prohibited except with permission in writing from the publisher. The CCC fee code is 0277$786 \mathrm{X} / 20 / \$ 21.00$.

Printed in the United States of America by Curran Associates, Inc., under license from SPIE.

Publication of record for individual papers is online in the SPIE Digital Library.

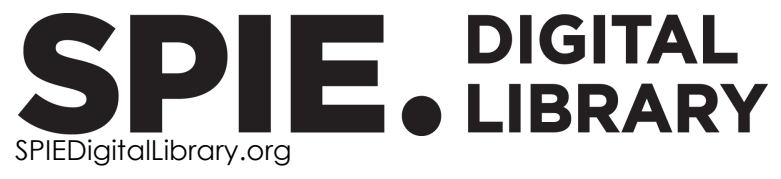

Paper Numbering: Proceedings of SPIE follow an e-First publication model. A unique citation identifier (CID) number is assigned to each article at the time of publication. Utilization of CIDs allows articles to be fully citable as soon as they are published online, and connects the same identifier to all online and print versions of the publication. SPIE uses a seven-digit CID article numbering system structured as follows:

- The first five digits correspond to the SPIE volume number.

- The last two digits indicate publication order within the volume using a Base 36 numbering system employing both numerals and letters. These two-number sets start with 00, 01, 02, 03, 04, $05,06,07,08,09,0 A, 0 B \ldots$ OZ, followed by 10-1Z, 20-2Z, etc. The CID Number appears on each page of the manuscript. 


\title{
Contents
}

\author{
vii Authors \\ ix Conference Committee \\ xi Introduction
}

STRUCTURED OPTICAL FIELDS, BEAM SHAPING, POLARIZATION CONTROL, PULSE SHAPING, FREQUENCY EXTENSION, AND ULTRAFAST LASER TECHNOLOGIES

1152202 Spin-orbit Laguerre-Gauss beam shaping (Invited Paper) [11522-1]

1152203 Photothermal energy conversion in plasmonic nano gap antennas: application to localized ZnO growth for nanophotonics [11522-8]

1152204 Molecular manipulation by plasmon induced optical force at solid-liquid interface under ambient condition [11522-9]

1152205 Ultrafast azimuth rotation of linearly polarized beam by use of a chirped optical pulse pair [11522-15]

1152206 Transmission spectral and diffraction pattern study on optical trapping and assembling of dielectric nanoparticles at solution/glass interface [11522-17]

1152207 Revisit of azimuthal doppler shift for transverse flow measurement by using an optical vortex beam [11522-21]

1152209 Spinning twin-mode generation in a bacteriorhodopsin suspension [1 1522-27]

$115220 \mathrm{~A}$ Correction to spatial mode transformation in a modified interferometer [11522-30]

11522 OB Isotopic hydrogen evolution reaction by plasmonic electrochemistry [11522-43]

$115220 \mathrm{C}$ Metal sphere separation in glass by the two laser beam illumination [11522-44]

11522 OD A general method for evaluation of effective Raman wavefront and phase shift in atom interferometry [11522-53] 
OPTICAL TRAPPING AND MANIPULATION, INCLUDING OPTICAL TWEEZER, HOLOGRAPHIC OPTICAL MANIPULATION, PLASMON TRAPPING, MULTI-PHOTON TRAPPING, AND ATOM TRAPPING AND COOLING

11522 OE Optical gradient force on Chiral nanoparticles [11522-3]

11522 OF Orbiting of dielectric particles around a single-mode ultrathin fiber waveguide [11522-4]

11522 OG Laser-heated microswimmers: optical manipulation of active particles (Invited Paper) [1 1522-6]

$11522 \mathrm{OH} \quad$ Manipulation of mica flakes using photothermally generated microbubbles [1 1522-7]

11522 Ol Vector beam induced mass transport in azo-polymer films [11522-12]

11522 0J A versatile fiber-optic platform for optical transport of solid particles and liquid droplets (Invited Paper) [1 1522-16]

11522 OK The trapping of a single chloroform microdroplet in water using optical tweezers [11522-18]

$11522 \mathrm{OL} \quad$ Optical micromanipulation using nonliner photo-responses [11522-19]

11522 OM Spatio-temporally-controlled synthesis of lead halide perovskite crystals by laser trapping [1 1522-25]

$11522 \mathrm{ON}$ Optical transport and sorting of fluorescent nanodiamonds inside a tapered glass capillary [11522-35]

1152200 Orbital analysis of single nanoparticle in-plane motion driven by an optical vortex [11522-38]

$11522 \mathrm{OP} \quad$ Observation of Mie scattering from a superconducting micro-particle [11522-39]

$115220 Q \quad$ Numerical simulation of micro- and nanoparticles orbital motion driven by an optical vortex [1 1522-40]

11522 OR Theory of optical response measurement of dimer molecules by photoinduced force microscopy [11522-41]

11522 OS Plasmon-induced transverse optical torque on nanostructures [11522-42]

11522 OT Fast iterative phase retrieval in holographic data storage [11522-47]

11522 OU Non interferometric phase retrieval in collinear holographic data storage [1 1522-48]

11522 OV Manipulation of CdSe/ZnS quantum dots in room-temperature fluid with an inhomogeneous electric field and optical excitation [1 1522-50] 
FUNDAMENTAL RESEARCH AND ADVANCED TECHNOLOGIES ENABLED BY STRUCTURED MATERIALS, SUCH AS METAMATERIALS, METASURFACES, AND PHOTONIC CRYSTALS

$11522 \mathrm{OW}$ Enhancement of electrocatalytic oxygen reduction reaction on Au-Ag nanorings by plasmon excitation [11522-10]

$115220 X \quad$ Spin/orbital angular momentum conversion at a nano scale (Invited Paper) [11522-11]

11522 OY Development of achromatic flat-surface gradient index micro lenses [11522-36]

$115220 Z$ Effect of photo-sensitizer and thermo-initiator concentrations on holographic recording performance of PQ/PMMA photopolymer [11522-49]

1152210 Observation of structural color in random Au nano-islands fabricated on dielectric nanopillars [11522-51]

1152211 Study on the causes of bubbles in PQ / PMMA optical holographic storage materials [11522-52]

ADVANCED DEVICES AND INSTRUMENTS, INCLUDING SPATIAL LIGHT MODULATOR, ADAPTIVE OPTICS, AND NEAR-FIELD OPTICAL DEVICES

$1152212 \quad$ Broadband terahertz single pixel imaging system [11522-31]

APPLICATIONS INCLUDING STRUCTURED MATERIAL PROCESSING, SINGLE MOLECULE TRAPPING, BIOPHOTONICS, METAMATERIALS, QUANTUM COMMUNICATIONS, AND SELECTIVE CONTROL OF CHEMICAL REACTION

1152213 Picosecond optical vortex induced chiral structures of azo-polymers via two photon absorption [11522-2]

$1152214 \quad$ Fractional optical vortex creates a curved "spin-jet" [11522-13]

1152215 Microscale perovskite crystal creation by optical vortex laser induced forward transfer [11522-14]

1152216 High-pressure microscopy for manipulating bionanomachines in living cells [11522-23]

1152217 Neuronal electrical activity induced by optical trapping of neurotransmitter receptors on neuron [11522-24]

1152218 Multidimensional spatial entanglement transfer through our existing fiber optic network [11522-32]

1152219 Silicon (111) chiral structures fabricated by illumination of optical vortex [11522-37]

$115221 \mathrm{~A}$ Controlling the propagation of surface plasmon polaritons by geometric phases [11522-45] 
NOVEL APPROACHES, INCLUDING NOVEL INTERACTION BETWEEN OPTICAL FIELDS AND MATERIALS ON NANO-SCALE, NOVEL REGIMES OF SPIN-ORBIT INTERACTION, AND QUANTUM CONTROL OF MOLECULAR DYNAMICS

11522 1B Quantum vortex visualization in superfluid helium [1 1522-5]

11522 1C Coherent oscillations of a birefringent microsphere in vacuum optical traps [11522-20]

11522 1D Bond-breaking and adsorption of molecules at plasmonic hot-spots initiated by infrared vibrational excitation [1 1522-33]

$11522 \mathrm{lE} \quad$ Fluorescence correlation spectroscopy for analysis of atto-liter space using three-dimensional super-resolution microscopy [11522-46]

INVITED SESSION OMC20

$115221 \mathrm{~F} \quad$ Light-induced biological waveguides (Invited Paper) [11522-26]

$115221 \mathrm{H} \quad$ Controlling orbital angular momentum in microscopic and topological systems (Invited Paper) [11522-29]

$1152211 \quad$ Enhanced optical trapping (Invited Paper) [11522-34] 


\section{Authors}

Numbers in the index correspond to the last two digits of the seven-digit citation identifier (CID) article numbering system used in Proceedings of SPIE. The first five digits reflect the volume number. Base 36 numbering is employed for the last two digits and indicates the order of articles within the volume. Numbers start with 00, 01, 02, 03, 04, 05, 06, 07, 08, 09, 0A, OB...0Z, followed by 10-1Z, 20-2Z, etc.

Ablez, Ablimit, 19

Ahn, Hyo-Yong, OE

Alvandi, Yousef, 01

Aoyagi, Shota, 1B

Arita, Yoshihiko, 1C

Asano, Riki, OV

Ashida, Masaaki, OP, 1B

Ashihara, Satoshi, 1D

Ayop, Shahrul Kadri, OK

Bae, Young-Gyu, 10

Bezryadina, Anna, IF

Biju, Vasudevanpillai, OM

Brasselet, Etienne, 02

Buczyński, Ryszard, OY

Butaite, Une G., 11

Chen, Xi, 0Z, 11

Chen, Yuxin, OZ, 11

Chen, Zhigang, $1 \mathrm{~F}, 1 \mathrm{H}$

Chiba, Akira, OC

Dholakia, Kishan, 1C

Doi, Kentaro, 00

Ferrer-Garcia, Manuel F., Ol

Filipkowski, Adam, OY

Forbes, Andrew, 18

Fujiwara, Hideki, 03

Fukuhara, Ryoma, OS

Gautam, Rekha, 1F

Gautam, Surya, OA

Gibson, Graham M., 11

Hansson, Tobias, $1 F$

Hao, Jianying, OT, OU

Hashiyada, Shun, OE

Hayasaka, Mizuki, OL

He, Jiahuan, 12

Hidai, Hirofumi, 0C

Himura, Haruhiko, 07

Ho, Ying-Lung D., 11

Hosokawa, Chie, 17

$\mathrm{Hu}$, Qingqing, OD

Huang, Zhiyun, $\mathrm{OZ}$

Iketaki, Yoshinori, $1 \mathrm{E}$

Imazeki, Taichi, OC

Ishihara, Hajime, OR

Ito, Syoji, OL

Kameyama, Tatsuya, OW

Kamit, Abdullah, 06

Karimi, Ebrahim, $\mathrm{Ol}$

Kasztelanic, Rafal, OY

Kawaguchi, Haruki, 14, 15
Kawano, Satoyuki, $00,0 Q$

Kim, Taehyun, 10

Kishimoto, Tatsunori, 17

Kitamura, Kyoko, 07

Kitazawa, Miki, 07

Koga, Masafumi, OL

Kokado, Kensuke, 1B

Kondo, D., OP

Kudo, Tetsuhiro, 06

Kudoh, Suguru N., 17

Kumakura, Mitsutaka, OP, OV

Kumar, Samir, $\mathrm{OH}$

Lamstein, Josh, $1 \mathrm{~F}$

Lee, Hyeonwoo, $0 \mathrm{~J}$

Lee, Jongsu, 10

Lee, Junhyung, 09

Lee, Seung-Yeol, 10

$\mathrm{Li}$, Yingying, OD

Lin, Feng, 1A

Lin, Xiao, OT, OU, OZ, 11

Litchinitser, Natalia M., OX

Liu, Jun, 18

Luo, Yukun, OD

Ma, Mingxiang, OD

Masuda, Keigo, 09, 13

Masuhara, Hiroshi, 06

Matsumura, Ryuta, $\mathrm{OH}$

Matsusaka, Souta, OC

Md Jahidul, Islam, OM

Minamimoto, Hiro, 04, OB

Minowa, Yosuke, 1B

Miyamoto, Katsuhiko, 09, 12, 13, 14, 15, 19

Miyamoto, Yoko, OA

Miyasaka, Hiroshi, OL

Mohamad Yusof, Mohd Farid, OK

Morandotti, Roberto, IF

Morichika, Ikki, ID

Morita, Noboru, OC

Morita, Ryuji, 05, 19

Moriwaki, Y., OP

Moriyasu, Takeshi, OV

Murakoshi, Kei, 04, OB

Murata, Takeshi, 09

Naik, Dinesh N., OA

Nakajima, Kichitaro, 00

Nakamura, Shinya, OL

Namura, Kyoko, $\mathrm{OH}$

Naoi, J., OP

Nape, Isaac, 18 
Nguyen, Hue Thi, OY

Nic Chormaic, Síle, OF

Nishiyama, Masayoshi, 16

Nobuhiko, Yokoshi, OR

Nozue, Goro, OV

Oh, Kyunghwan, 0J

Ohno, Seigo, 12

Oka, Kazuhiko, 05

Okada-Shudo, Yoshiko, 09

Okamoto, Hiromi, OE

Omatsu, Takashige, 05, 09, 12, 13, 14, 15, 19

Ookawa, Taisei, 12

Otsuka, Ryohei, ON

Oyamada, Nobuaki, 04

Park, Junbum, 0J

Perez, Nicolas, $1 \mathrm{~F}$

Phillips, David B., 11

Pin, Christophe, 03, 0N

Preece, Daryl, $1 \mathrm{H}$

Pysz, Dariusz, OY

Ren, Yuhong, OT, OU

Rubinsztein-Dunlop, Halina, $1 \mathrm{H}$

Ryu, Yong-Sang, 10

Samlan, C. T., OA

Sasaki, Keiji, 03, ON

Sasaki, Rin, 05

Sasaki, S., OP

Sasamoto, Kosuke, OW

Sato, Daiki, OB

Setoura, Kenji, OL

Shi, Zhiwei, $1 \mathrm{H}$

Shimomura, Takayuki, OV

Shimura, Tsutomu, OS

Shinada, Masataka, 09

Shiraishi, Ami, 13

Simpson, Stephen H., $1 \mathrm{C}$

Song, Haiyang, $0 \mathrm{Z}, 11$

Sotome, Hikaru, OL

Stepien, Ryszard, OY

Sun, Jingbo, ox

Suzuki, Motofumi, $\mathrm{OH}$

Suzuki, Tatsuro, 03

Taghizadeh, Mohammad R., OY

Taguchi, Takahisa, 17

Takanune, M., OP

Tan, Xiaodi, OT, OU, 0Z, 11

Tanaka, Yoshito, OS

Taverne, Mike, 11

Taylor, Jonathan M., 11

Tkachenko, Georgiy, OF

Toda, Yasunori, 05

Toftul, Ivan, OF

Torimoto, Tsukasa, OW

Toyoda, Kohei, 09

Truong, Viet Giang, OF

Tseng, Ching-Shiang, 06

Tsuji, Tetsuro, $0 Q$

Tsujimura, Tempei, 00

Umesato, Kei, 14, 15

Vallés, Adam, 12, 18
Viswanathan, Nirmal K., OA

Waddie, Andrew, OY

Wang, Jian, 18

Wang, Jie, OD

Wang, Kun, OT, OU

Wang, Qianke, 18

Wetzel, Benjamin, IF

Wu, Chenhao, $\mathrm{OZ}$

Würger, Alois, OG

Xiang, Yinxiao, If

$X U$, Fufang, OD

Yamamoto, Kiroku, OV

Yamane, Hidemasa, OR

Yamane, Keisaku, 05, 14

Yamanishi, Junsuke, OE

Yoshizawa, Taiki, 09

You, Jie, OD

Yu, Eui-Sang, 10

Yuyama, Ken-ichi, 0M, 15

Zemánek, Pavel, 1C

Zhan, Yingwen, 0 l

Zhang, Yuanyin, $\mathrm{OZ}$

Zhao, Yang, IA 


\title{
Conference Committee
}

\author{
Conference Chair
}

Takashige Omatsu, Chiba University (Japan)

\section{Conference CoChairs}

Kishan Dholakia, University of St. Andrews (United Kingdom)

Hajime Ishihara, Osaka Prefecture University (Japan)

Keiji Sasaki, Hokkaido University (Japan)

\section{Conference Program Committee}

Masaaki Ashida, Osaka University (Japan)

Satoshi Ashihara, The University of Tokyo (Japan)

Yung-Fu Chen, National Chiao Tung University (Taiwan)

Síle Nic Chormaic, Okinawa Institute of Science and Technology

Graduate University (Japan)

Yasuyuki Kimura, Kyushu University (Japan)

Kyoko Kitamura, Kyoto Institute of Technology (Japan)

Yuichi Kozawa, Tohoku University (Japan)

Yoko Miyamoto, The University of Electro-Communications (Japan)

Ryuji Morita, Hokkaido University (Japan)

Kei Murakoshi, Hokkaido University (Japan)

Kyoko Namura, Kyoto University (Japan)

Kyunghwan Oh, Yonsei University (Korea, Republic of)

Seigo Ohno, Tohoku University (Japan)

Hiromi Okamoto, Institute for Molecular Science (Japan)

Ichiro Shoji, Chuo University (Japan)

Yasuhiro Sugawara, Osaka University (Japan)

Yasuyuki Tsuboi, Osaka City University (Japan) 
Proc. of SPIE Vol. 11522 1152201-10

Downloaded From: https://www.spiedigitallibrary.org/conference-proceedings-of-spie on 25 Apr 2023 Terms of Use: https://www.spiedigitallibrary.org/terms-of-use 


\section{Introduction}

Since the first demonstration of an optical tweezer based on optical radiation forces (scattering and gradient forces) created by a tightly focused laser beam, optical tweezers have been widely investigated in a variety of research fields, including biology, physics, and chemistry. In fact, Dr. A. Ashkin was awarded Nobel Prize in Physics, for contributing to a pioneering work of optical manipulation, 2018.

Conventional optical tweezers have been mostly adopted to dielectric particles with a dimension range from hundreds of nanometers to tens of micrometers. However, they do not always enable us to efficiently trap nanosacle-sized dielectric particles as well as metallic particles.

In recent years, plasmonic tweezers based on enhanced radiation forces owing to surface plasmon polaritons in metallic nanostructures have been successfully demonstrated to efficiently trap and manipulate both nanosacle-sized dielectric and metallic particles.

Also, structured lights, such as higher order Laguerre-Gaussian and Bessel beams carry optical angular momenta, and they provide unique tweezing abilities, for instance, for inducing an orbital motion of the trapped particles without employing mechanical systems.

Since 2014, the OMC has been successfully collecting more than 80 participants from home and abroad. The OMC 2020 conference aims to present and discuss up-to-date scientific subjects, new technologies, and applications related to the fields of optical and plasmonic tweezers, the manipulation of nanostructures, structured optical fields and their satellite topics.

\section{Takashige Omatsu Kishan Dholakia Hajime Ishihara Keiji Sasaki}


Proc. of SPIE Vol. 11522 1152201-12

Downloaded From: https://www.spiedigitallibrary.org/conference-proceedings-of-spie on 25 Apr 2023 Terms of Use: https://www.spiedigitallibrary.org/terms-of-use 